\title{
A SELF-CONSISTENT MODEL FOR CLEAVAGE IN THE PRESENCE OF PLASTIC FLOW
}

\author{
G. E. BELTZ', J. R. RICE', C. F. SHIH ${ }^{3}$ and L. XIA ${ }^{3}$ \\ 'Department of Mechanical and Environmental Engineering, University of California. Santa Barbara, \\ CA 93106-5070. 'Division of Engineering and Applied Sciences and Department of Earth and Planetary \\ Sciences, Harvard University, Cambridge, MA 02138 and 'Division of Engineering. Brown University. \\ Providence. RI 02912, U.S.A.
}

(Received 4 October 1995; in retised form 23 January 1996)

\begin{abstract}
A theory is proposed for cleavage in the presence of plastic flow. in circumstances which do not involve strong viscoplastic retardation of dislocation motion. We build upon recent notions that recognize the large disparity between relevant length scales involved in plastic flow processes around cracks in metals and on metal-ceramic interfaces. The lengths consist of (1) the Burgers vector, (2) the nominal dislocation spacing. (3) the elastic "cell" dimension, and (4) the overall plastic zone size. Of particular interest is the phenomenon of "brittle" crack growth in the presence of pre-existing. apparently mobile. dislocations, which has been observed in several material systems. A continuum elastic-plastic finite element model is utilized that assumes the presence of a dislocation-free strip of elastic material of height $D$ surrounding a crack tip, from which dislocations are assumed not to emit. The parameter $D$ is self-consistently chosen by identifying a maximum equivalent Mises stress in the plastic zone with that predicted by a phenomenological strength law of the type first used by Taylor and Orowan. in which strength varies inversely with nominal dislocation spacing or with cell size, either of which is identified as $D$ in different interpretations of the model. For steady-state crack growth to occur, it is found that the applied energy release rate $G$ must generally be several orders of magnitude larger than the ideal work necessary to separate the interface, at least when $D$ is taken as dislocation spacing. Furthermore, this "shielding" ratio is found to be strongly sensitive to the ideal work of fracture itself, as well as other material properties. Copyright $\subseteq 1996$ Acta Metallurgica Inc.
\end{abstract}

\section{INTRODUCTION}

The mechanical behavior of structural materials is governed by processes occurring over a diverse range of length scales. A specific material model, though, usually focuses only on a limited range of length scales. Continuum models based on elastic and/or plastic constitutive relations, for example, are appropriate for analyses of the macro/micro-scopic mechanical response of solids, but do not accurately represent the underlying crystal lattice and its associated defects, such as dislocations and grain boundaries. At another level, discrete dislocation modeling may capture many of the details of a crystal's nanoscopic mechanical response, but is limited to the consideration of nominally elastic behavior (i.e. relatively small or non-existent dislocation densities). This multiple-scale approach to materials engineering has led to a wealth of understanding within each model's domain of applicability. Nevertheless, many problems exist for which interaction between length scales is of central importance. Recent consideration of the competition between cleavage decohesion and dislocation emission at a crack tip, and its relation to ductile vs brittle behavior, exemplifies this duality [1].

In this paper, we build upon a recent model by Suo et al. [2] that recognizes the large disparity between relevant length scales involved in plastic flow processes around cracks in metals and metal-ceramic interfaces. These consist of (1) the Burgers vector, $b \sim 10^{-10} \mathrm{~m}$, (2) the nominal dislocation spacing $1 / \sqrt{\rho_{\text {disl }}}$, where $\rho_{\text {dist }}$ is the dislocation density, (3) the elastic cell size within the dislocation structure, $D_{\text {cetl }} \sim 10^{-6} \mathrm{~m}$, and (4) the overall plastic zone size, which scales approximately as $\left(K / \sigma_{0}\right)^{2}$, where $K$ is a stress intensity factor and $\sigma_{0}$ is the yield stress. Of particular interest is the phenomenon of crack growth in the presence of pre-existing dislocations, which has been observed in several systems. For example, a sharp crack can propagate along a gold-sapphire interface [3]; nevertheless, the gold deforms plastically, indicating the presence and motion of vast quantities of dislocations. Furthermore, the measured fracture energy is orders of magnitude larger than the adhesive energy; when the interface contains carbon, a segregant that has been estimated to reduce the adhesive energy by about a factor of 2 , the measured fracture energy is reduced by nearly an order of magnitude but is still significantly larger than the adhesive energy [3]. Other examples include copper bicrystals contaminated with bismuth [4], copper-sapphire interfaces [5], copper-glass [6], and niobium-alumina $[7,8]$. 
A large literature exists on the modeling of cracks in plastically deformable materials, both from continuum and atomistic standpoints. However, the material behavior described above has eluded a reasonable mechanistic explanation (see Ref. [9] and references therein) except perhaps in cases for which strong viscoplastic resistance to plastic flow, i.e. strongly limited dislocation mobility, is present at deformation rates prevailing over distances of, at least, a few dislocation spacings near the crack tip. The latter includes situations of dynamic crack growth in a visco-plastic continuum, sometimes begun through rapid "crack injection" by failure of a brittle phase (e.g. carbide in steel), as investigated by Mataga et al. [10], Hart [11] and Jokl et al. [12, 13]. Similar consideration of these effects at the discrete dislocation scale have been examined by Huang and Gerberich [14] and Hirsch and Roberts [15]. We believe that other cases of cleavage in the presence of high dislocation densities, but without the assistance of strong viscoplastic resistance to flow, might be tied to the interplay between processes occurring over many length scales that naturally arises when plasticity is regarded as a discrete or granular phenomenon. We let $D$ denote a characteristic spacing of barriers to dislocation motion. Then, in the one hand, continuum plasticity, whether it be for isotropic materials or single crystals, is only rigorously valid when the stress variation over a multiple of $D$ is small compared to the macroscopic yield strength. In other words, the theory takes for granted that the relevant length scales of interest are large enough so that the medium is free to deform, without concern for the dislocation motion through barriers with length scale $D$ that actually is responsible for plastic deformation. On the other hand, processes occurring between length scales $b$ and $D$ are extremely important as well; an example is dislocation nucleation at a crack tip and its relation to the continued stability of the sharp crack [16].

Pre-existing dislocations are inevitably present in abundance in all metals, and to some extent most non-metals, except for some prepared under very precise conditions (as silicon for electronic devices). It is not commonly realized that at a load level great enough to meet conditions for either Griffith cleavage or dislocation nucleation at a crack tip in a metal crystal, the corresponding concentrated stress field near the crack tip contains large enough shear stresses to move dislocations over distances that are, typically, many times the spacings of those pre-existing dislocations. Thus, it is very easy to move pre-existing dislocations near a crack tip in metals, and that is usually expected to occur well before the critical loading to either nucleate a dislocation at a crack tip or cause Griffith cleavage. Indeed, much of the empirical evidence that sometimes leads to the conclusion that dislocations nucleate at a crack tip at very low load levels [17] may simply signify that pre-existing dislocations have been activated.
To understand the point further. note that the standard elastic mode I stress field creates a Mises effective stress $\sigma_{\text {eff }}$ [evaluated approximately as $\sqrt{3\left(\sigma_{v}-\sigma_{w}\right)^{2 / 4}+3 \sigma_{x v}^{2}}$ here] near the tip given as

$$
\sigma_{\mathrm{err}}=\frac{S(\theta) K_{\mathrm{ur}}}{\sqrt{r}}=S(\theta) \sqrt{\frac{4 \mu_{r_{\text {int }}^{\prime}}}{(1-v) r}},
$$

where the stress intensity factor $K_{\mathrm{tun}}$ is taken as its Griffith value, i.e. $G_{\text {tip }} \equiv(1-v)\left(K_{\text {tip }}\right)^{2} / 2 \mu=2 ;$ inu, with $2 \gamma_{\text {ins }}$ being the ideal work of fracture of the lattice plane or grain interface considered. Also, $\mu$ is the shear modulus and $v$ the Poisson ratio, and

$$
S(\theta)=\frac{|\sin \theta|}{2} \sqrt{\frac{3}{2 \pi}},
$$

with $\theta=0$ on the prolongation of the crack plane. We wish to know over what size in the material is $\sigma_{\text {eff }}$ greater than the yield stress $\sigma_{0}$. Now, to the neglect of a lattice resistance part of $\sigma_{0}$ that we call $\sigma^{*}(T)$ below, it may be assumed that $\sigma_{0}$ scales inversely with the spacing $D_{0}$ of barriers to dislocation motion, $\sigma_{0}=\alpha_{1} E b / D_{0}$, as in phenomenological strength laws introduced by Taylor [18] and Orowan [19]. Here $E$ is Young's modulus and, as explained in Section 4. the parameter $\alpha_{1}$ ranges from 0.4 to 0.8 if we identify $D_{0}$ as the nominal dislocation spacing $1 / \sqrt{\left(\rho_{\text {disl }}\right)_{0}}$ before deformation begins. Alternatively, $\alpha_{t}$ ranges from 5 to 10 if we identify $D_{0}$ as the initial dislocation cell size, such being well defined at least at rather large pre-strains. Taking $\theta=90^{\circ}$ and $v=0.3$, we therefore find that at the Griffith load considered the yield stress is exceeded $\left(\sigma_{\text {eff }}>\sigma_{0}\right)$ over a distance $r$ given by

$$
\frac{r}{D_{0}}=0.10 \frac{\gamma_{\text {int }} / \mu b}{\alpha_{1} \sigma_{0} / E}=0.26 \gamma_{\text {int }} / \alpha_{1} \sigma_{0} b
$$

For metal crystals $\gamma_{\text {int }} / \mu b$ ranges from 0.05 to 0.25 (Table 1 ) and thus, if we identify $D_{0}$ as $1 / \sqrt{\left(\rho_{\text {disi }}\right)_{0}}$ and therefore take $\alpha_{t}=0.6$, and consider metals with yield strength $\sigma_{0}=10^{-3} E$, then $r$ is estimated as $8-40 D_{0}$. That is, there is ample stress at Griffith conditions to move quite a few dislocations in materials of such a strength level, and the model which we develop here is consistent with the empirical evidence that their motion and shielding of the tip greatly increases the load level for cleavage. Equation (3) also introduces the combination of material properties $\gamma_{\text {in }} / \alpha_{1} \sigma_{0} b$, which we shall see to be an important parameter for characterizing the intensity of plastic shielding of a brittle crack tip.

A major conceptual problem with bringing together continuum and atomistic theories of fracture is intimately tied to differences in the various characteristic stresses: the atomic cleavage strength is known to be orders of magnitude larger than the macroscopic yield strength. However, if the ratio between the two is assumed to be larger than about 4 , as in a Barenblatt-like decohesion model within the 
Table 1. Elastic core parameters for simple model of Section 2 and Figs 1 and 2. for cleavage at a non-emitting crack tip in a plastically deformable material. These values are based on $v=0.3 . x=0.55$ and $S(\theta)=\left(3 / 2 \pi^{3}\right)^{12}$ (its average over $\theta$ ). with the exception of the final column. $\sigma^{*}$ is assumed to be zero. (Materials with the larger values of $\gamma m / \mu b$ may not actually be consistent with the assumption of a

\begin{tabular}{|c|c|c|c|c|}
\hline System & $\operatorname{in} / \mu b$ & riors: $h$ & $\sigma_{\text {come }} / E$ & $\sigma_{i z=1}^{*} / E$ \\
\hline \multicolumn{5}{|l|}{ Ductile metals: } \\
\hline $\mathrm{Li}$ & 0.26 & 28 & 0.0195 & 0.0047 \\
\hline $\mathrm{Au}$ & 0.23 & 32 & 0.0173 & 0.0041 \\
\hline $\mathrm{Al}, \mathrm{Cu}$ & 0.17 & 44 & 0.0128 & 0.0031 \\
\hline $\mathrm{Ni}$ & 0.12 & 62 & 0.0090 & 0.0022 \\
\hline $\mathrm{Nb}$ & 0.11 & 67 & 0.0085 & 0.0020 \\
\hline $\mathrm{Ta}, \mathrm{V}$ & 0.10 & 74 & 0.0075 & 0.0018 \\
\hline \multicolumn{5}{|l|}{ Borderline: } \\
\hline $\mathrm{Fe}$ & 0.079 & 94 & 0.0059 & 0.0014 \\
\hline \multicolumn{5}{|l|}{ Brittle metals: } \\
\hline lr & 0.057 & 130 & 0.0043 & 0.0010 \\
\hline Cr. W & 0.040 & 185 & 0.0030 & 0.00072 \\
\hline Mo & 0.036 & 205 & 0.0027 & 0.00065 \\
\hline $\begin{array}{l}\text { Fe grain boundary } \\
\text { with } \mathrm{S} \text { or } \mathrm{P} \\
\text { segregation }\end{array}$ & $0.06-0.07$ & $105-123$ & $0.0045-0.0053$ & $0.0011-0.0013$ \\
\hline $\begin{array}{l}\text { Metal-cermaic } \\
\text { interface } \\
\text { (e.g. } \mathrm{Cu} / \mathrm{Al}_{2} \mathrm{O}_{3} \text { ) }\end{array}$ & $0.02-0.03$ & $247-370$ & $0.0015-0.0023$ & $0.00036-0.00054$ \\
\hline
\end{tabular}

framework of continuum plasticity, ductile response only with no possibility of cleavage is predicted [20-23]. The model by Suo et al. [2] built-in the assumption of a dislocation-free region of material surrounding a crack tip, from which dislocations are assumed not to emit. Moreover, we proceed with the understanding that the parameter $D$ characterizes the size of a nominally-elastic zone whose evolution may include the nucleation of dislocations (from noncrack tip sources) that subsequently become incorporated into the plastic zone. Having made these assumptions, a length $D$ over which only elastic stresses operate is built into the model, and hence the restriction that the ratio of atomic cleavage strength to yield stress be no greater than about 4 should (in principle) not be present. The length $D$ may be said to correspond physically to a so-called dislocation free zone (DFZ). although such terminology is also used to indicate a zone surrounding an emitting crack tip through which dislocations may pass but which is otherwise dislocation free [24].

Figure 1 illustrates how characteristic stresses are expected to vary by orders of magnitude as a crack tip is approached. The competition between atomic decohesion and dislocation nucleation is not addressed in the current theory; we simply assume that the tip does not emit dislocations in the cases for which the theory is intended. The assumption of a non-emitting tip is certainly justified for materials of relatively low $\gamma_{\text {int }} / \mu b[1,25]$ or in the presence of contaminated grain boundaries. Even then, it is at best only an approximation, in that conditions for emission are strongly sensitive to superposition of small amounts of mode II or III shear on a mode I crack tip [1], so that perturbations from nominally tensile loading (due to misalignment of local cleavage planes to the loading direction, to steps on the crack surface or to asymmetries in the pre-existing dislocation distribution relative to the crack plane) may cause emission events at an otherwise non-emitting tip.

Whereas the model of Suo $e t$ al. [2] left the elastic strip height $D$ as an adjustable parameter, the current work introduces a self-consistent determination of $D$, regarding it as the spacing of barriers to dislocation

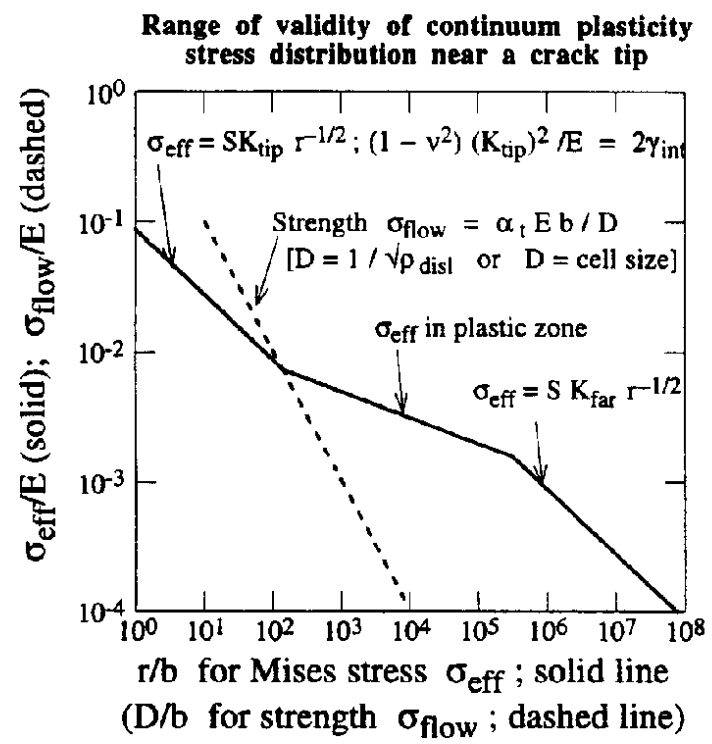

Fig. 1. Conceptual plot of the large stress variation ahead of a sharp crack tip in a plastically deforming metal. Indicated are: (1) the elastic core region, characterized by a classical linear elastic stress distribution with $K=K_{\text {tip }}$ equated to value for Griffith cleavage; (2) continuum plastic stress distribution; and (3) outer elastic zone with $K=K_{\text {fur }}$. 
motion in the strain hardened state prevailing immediately adjacent to the crack tip. That is, we insist that the strip height $D$ be chosen so that the flow strength given by equation (4) below, evaluated with that same $D$, is equal to the maximum $\sigma_{\text {eff }}$ calculated according to continuum plasticity in the plastic zone adjoining the strip. The model is described in more detail in the following three sections.

\section{ELEMENTARY ESTIMATES FOR CLEAVAGE ACCOMPANIED BY PLASTIC FLOW}

The plot in Fig. 1 conveys the essential physical motivation for the model discussed in this paper. We begin in this section by making some simple (and. as will be seen, not so accurate) estimates using a variant of a similar line of reasoning given earlier by Thomson [26]. At small distances from a stressed crack (i.e. $r \ll r_{\text {corc }}$, where $r_{\text {core }}$ is characteristic of a dislocation-free region about the non-emitting tip), stresses would be expected to vary according to equation (1).

A pertinent quantity is the length scale beyond which the stresses in the crack tip plastic zone will be suitably described by continuum plasticity. We may write the flow strength, $\sigma_{\text {fow }}$ within the plastic zone as being tied to the dislocation barrier spacing $D$ through the relation

$$
\sigma_{\text {fow }}=\alpha_{\mathrm{t}} E b / D+\sigma^{*}(T),
$$

where now $D$ is reduced from its initial value $D_{0}$ at first yielding, reflecting dislocation generation and hardening in the plastic flow, and we note that there may be an additional part of the flow strength. $\sigma^{*}(T)$, that is not related to dislocation spacings and instead reflects an intrinsic lattice resistance. Again, one may consider two interpretations, with $D$ as the nominal dislocation spacing $1 / \sqrt{\rho_{\text {disl }}}$ or as the dislocation cell size, and the respective ranges of $x_{1}$ are given earlier. As suggested by the notation, we consider $\sigma^{*}(T)$ to be temperature-dependent; it is expected to be negligibly small for f.c.c. metals, and monotonically decreasing with temperature for other crystals. In fact, $\sigma^{*}$ also depends on strain rate but we assume here that such a dependence is small, our aim being to explore explanations of brittleness that do not rely on limited dislocation mobility and strong viscoplastic effects like in Refs [10-13].

It is reasonable to assert that continuum plasticity is a consistent theory only at size scales $r$ from the tip that are greater than the barrier spacing $D$ involved in the flow process; that is, at distances $r$ corresponding to values of $\sigma_{\text {efr }}$ predicted by a continuum plasticity solution (solid line, Fig. 1) that lie above and to the right of the dashed-line plot of equation (4) in Fig. 1 (for the case $\sigma^{*}=0$ ). Material points closer than the intersection of those lines are closer to the crack tip than the barrier spacing in the plastic flow process. We describe that intersection distance as $r_{\text {core }}$, so that the point of intersection corresponds, in this approximate analysis, to $r=D=r_{\text {core }}$.

Now, if we assume that equation (1), which necessarily applies very close to a non-emitting crack tip at its cleavage load, also applies out to the extremities of the elastic core at the crack tip, then we can estimate $r_{\text {core }}$ by setting $\sigma_{\text {efl }}$ of equation (1). evaluated at $r=r_{\text {core }}$, equal to $\sigma_{\text {fios }}$ of equation (3) evaluated at $D=r_{\text {core }}$. Calling the common value of the stresses $\sigma_{\text {core }}$, we can therefore solve to obtain (in the case of $\sigma^{*}=0$ ) that

$$
\frac{r_{\mathrm{core}}}{b}=\frac{\left(1-v^{2}\right)(1+v) x_{i}^{2}}{S^{2}(\theta)}\left(\frac{\mu b}{\gamma_{\mathrm{imt}}}\right)
$$

and

$$
\frac{\sigma_{\mathrm{core}}}{E}=\frac{S^{\prime}(\theta)}{\left(1-v^{2}\right)\left(1+v^{\prime}\right) \alpha_{1}}\left(\frac{\gamma^{\prime} \mathrm{mt}}{\mu b}\right)
$$

These estimates of $r_{\text {core }}$ and $\sigma_{\text {core }}$ are tabulated in Table 1 for values of the dimensionless material ratio $\gamma_{\text {int }} / \mu b$ characteristic of a range of metals (ductile to brittle), iron with contaminated grain boundaries, and the $\mathrm{Cu}-\mathrm{Al}_{2} \mathrm{O}_{3}$ interface, all for $\sigma^{*}=0$. The pure metal $\gamma_{\text {im }}\left(=\gamma_{s}\right)$ values are from the tabulation in Ref. [1], $b$ is for a full (versus partial, for f.c.c.) dislocation. $v$ and $x_{t}$ have been taken as 0.3 and 0.55 , respectively. and $S(\theta)$ has been taken as its average over $\theta$, $\left(3 / 2 \pi^{3}\right)^{1: 2}$. Additionally, the stress in the elastic core as well as the flow strength given by equation (4) are plotted in Fig. 2(a). The results suggest that the core is large enough, at least in certain metals or systems. to justify the use of a continuum elastic theory within that region (if one accepts the fact that continuum mechanics is valid for any length scale greater than a "few" atomic spacings). Furthermore, the basic calculation admits the possibility of attaining the Griffith cleavage load for a non-emitting crack tip embedded in a large plastic zone, assuming that the material can be strain hardened up to stress levels like $\sigma_{\text {core }}$ without the required strains being so large that the crack tip region is deformed (at the continuum scale) into a rounded notch. We will subsequently consider this failure mechanism by more suitably carrying out the matching of continuum elastic and elastic-plastic fields, using finite element solutions, in a scheme that will additionally account for growing cracks in ductile materials.

We may note, however, that this simple analysis suggests that even if materials of relatively larger $\gamma_{\mathrm{n}} / \mu b$ are assumed to have non-emitting tips (in fact, the estimates of Refs $[1,25]$ suggest that only materials of lower $\gamma_{\text {ini }} / \mu b$ will actually be non-emitting), the amount of hardening to reach the required $\sigma_{\text {core }}$ values found is so unrealistically large that, for all practical purposes, those materials could not cleave. In that sense there is consistency between the atomic scale $[1,25]$ and present macroscopic plastic scale considerations of brittle versus ductile response. 
To gain insight into the important effect of temperature on such calculations, we assume that the overall temperature dependence of the flow stress enters through $\sigma^{*}$. For $\sigma^{*} \neq 0$, equations (5) generalize to the following root in the denominator of equation $(6 \mathrm{a})$ becomes non-positive, i.e. when

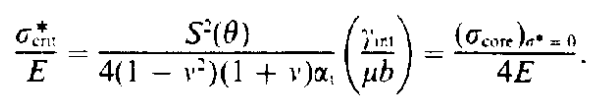

$$
\begin{aligned}
& \frac{r_{\text {cort }}}{b}=\frac{4\left(1-v^{2}\right)(1+v) \alpha_{i}^{2}}{S^{7}(\theta)\left(1+\sqrt{1-\frac{4\left(1-v^{2}\right)(1+v) \alpha_{1}}{S^{2}(\theta)}\left(\frac{\mu b}{\gamma_{\mathrm{m}}}\right)\left(\frac{\sigma^{*}}{E}\right)}\right)^{2}}\left(\frac{\mu b}{\gamma_{\text {int }}}\right) \\
& \frac{\sigma_{\text {core }}}{E}=\frac{S^{2}(\theta)}{2\left(1-v^{2}\right)(1+v) \alpha_{1}}\left(\frac{\gamma \text { int }}{\mu b}\right)\left(1+\sqrt{1-\frac{4\left(1-v^{2}\right)(1+v) \alpha_{\mathrm{t}}}{S^{2}(\theta)}\left(\frac{\mu b}{\gamma_{\text {int }}}\right)\left(\frac{\sigma^{*}}{E}\right)}\right) .
\end{aligned}
$$

Suppose that $\sigma^{*}$ gradually increases as temperature decreases. Then, according to this simple model, there exists a critical value $\sigma_{c r i 1}^{*}$, above which there is fracture without a macroscopic plastic zone. The crossover occurs when the argument to the square-

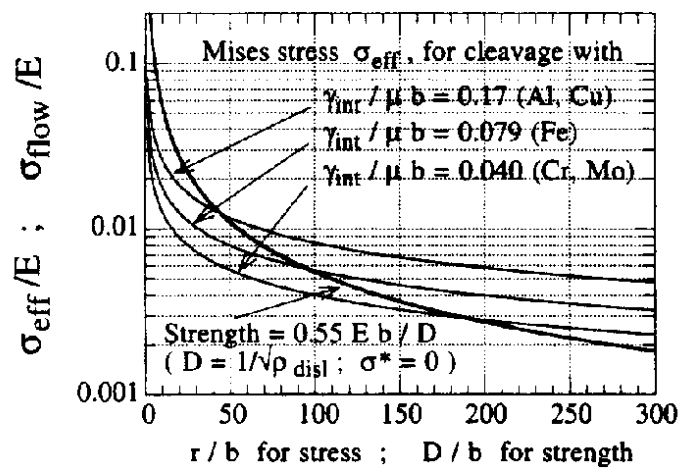

(a)

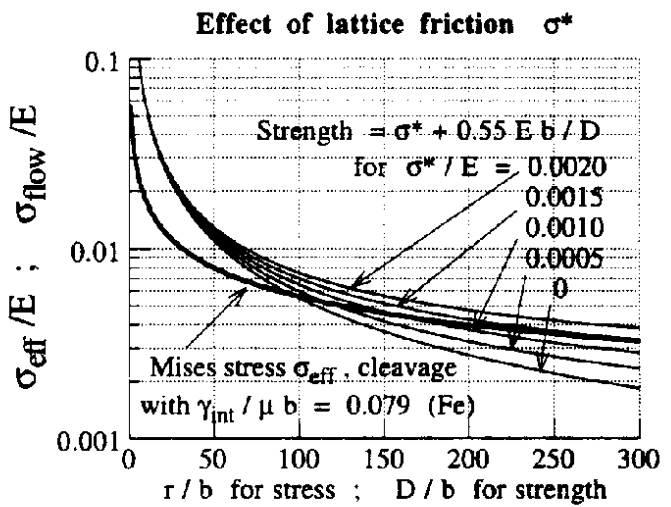

(b)

Fig. 2. (a) Critical stress distribution ahead of a crack tip for several values of $i$ im $i \mu b$, as well as flow stress as a function of elastic length $D$. For $r<r_{\text {core }}$ (the crossover between two types of curves above), the elastic core field is expected to dominate. (b) Stress and strength for the case of $\gamma_{\mathrm{im}} / \mu b$ (appropriate for $\mathrm{Fe}$ ) and several values of $\sigma^{*}$, showing the strong effect upon $r_{\text {corr }}$.
Graphically, the sharp onset of the effect can be seen in Fig. 2(b), where strength has been plotted for several values of $\sigma^{*}$, with other parameters estimated for $x$-iron. In this case $\sigma_{\text {crit }}^{*} \approx 0.0014 E$. Numerical values of the critical $\sigma^{*}$ for other materials are tabulated in Table 1. Using very similar arguments, Ashby and Embury [27] have proposed that for a given value of the lattice resistance, there exists a critical density of dislocations at the crack tip (i.e. a critical value of $\left.1 / D^{2}\right)$. below which the crack would run without interacting with dislocations. They thus showed that such a mechanism is a viable explanation for the common observation that plastic working lowers the brittle-to-ductile transition temperature in several b.c.c. metals.

\section{A FINITE ELEMENT MODEL FOR STEADY-STATE CLEAVAGE ACCOMPANIED BY PLASTICITY}

In order to make more quantitatively reliable predictions for a growing crack, assumptions must be made regarding the shape of the elastic core. As introduced by Suo et al., the strip model shown in Fig. 3(b) is adopted. Consider a cleavable, rate-independent, elastic-plastic material with Griffith energy $2 \gamma_{\text {int }}$ (which reduces to twice the surface energy for homogeneous materials), yield strength $\sigma_{0}$, Young's modulus $E$ and strain-hardening coefficient $N$. Material outside the strip is permitted to yield following the standard Prandtl-Reuss isotropically hardening continuum plasticity model, based on the second invariant of deviatoric stress. The true-stresslogarithmic strain curve in uniaxial tension. which generalizes in terms of Mises equivalent values, is specified by

$$
\begin{gathered}
\varepsilon=\frac{\sigma}{E} \quad \sigma<\sigma_{0} \\
\varepsilon=\frac{\sigma_{0}}{E}\left(\frac{\sigma}{\sigma_{0}}\right)^{1 N} \quad \sigma \geqslant \sigma_{\|} .
\end{gathered}
$$

Material within the strip remains elastic. The major weakness of the model is that the elastic strip 
is an uncertain proxy for the actual sort of elastic core at the tip envisioned in Fig. 3(a). Although some calculations have been carried out for a stationary crack tip embedded in a circular elastic zone [28, 29], the strip model retains the feature that steadl-state crack growth may be straightforwardly modelled with existing FEM codes. The mesh used for this model is shown in Fig. 4. Consider a scenario whereby the system, containing the stationary crack, is loaded monotonically. The external loading parameter may be specified as $G_{\text {far }}$. In certain situations, $G_{\text {far }}$ may differ from the crack tip energy release rate, $G_{\text {tip }}$ : however, the configuration possesses material properties which are homogeneous in the crack growth direction, hence arguments may be invoked in connection with the $J$ integral [30] to prove that $G_{\text {tip }}=G_{\text {fur }}$ for the stationary crack case, to within the usual approximation in such considerations of a "deformation plasticity" interpretation of the stationary crack field.

Under the assumptions presented thus far, the crack will begin to grow when $G_{\mathrm{far}}=G_{\mathrm{tp}}=2 \gamma_{\mathrm{int}}$. Independent results by Mesarovic [31] have suggested that, due to interactions between the crack and near-tip dislocations swept in from farther distances, an effective value of $2 \%$ int (greater than but similar in magnitude to the Griffith level) ought to be identified

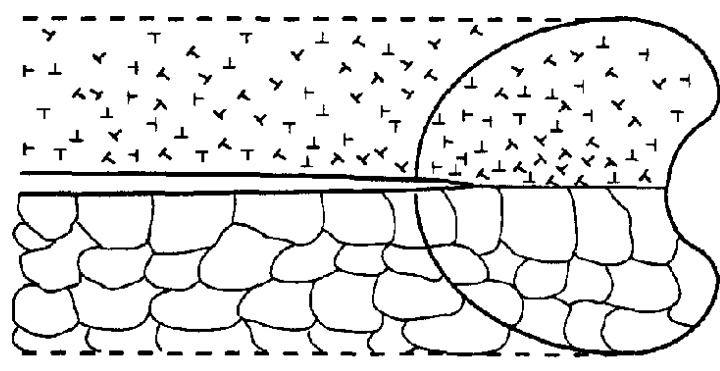

(a)

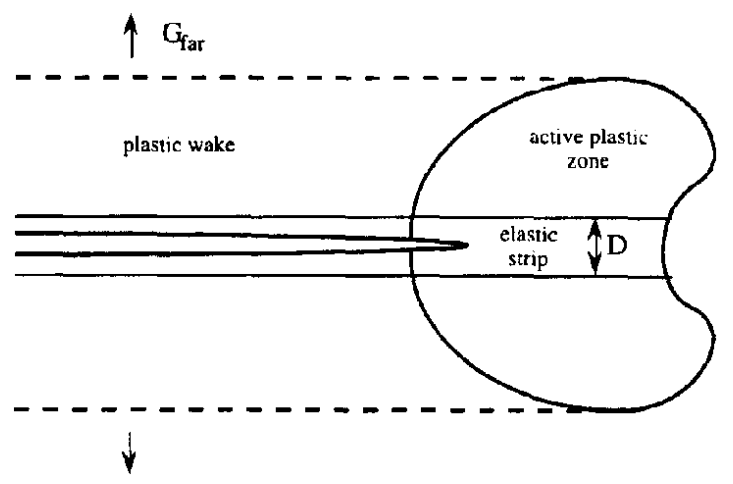

(b)

Fig. 3. (a) Schematic of a crack growing in steady-state through an elastic-plastic material, showing the active plastic zone, the plastic "wake" (elastic unloading), and possible dislocation arrangements characteristic of plastic flow processes. (b) Elastic strip configuration used in this note to model the situation in (a).

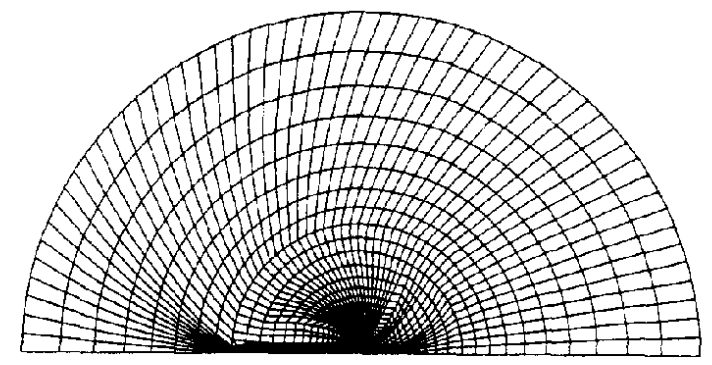

(a)

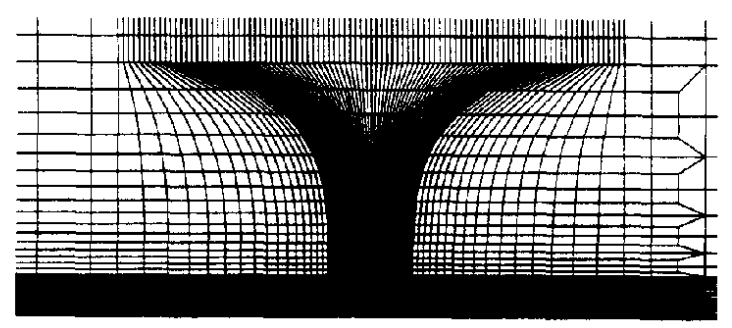

(b)

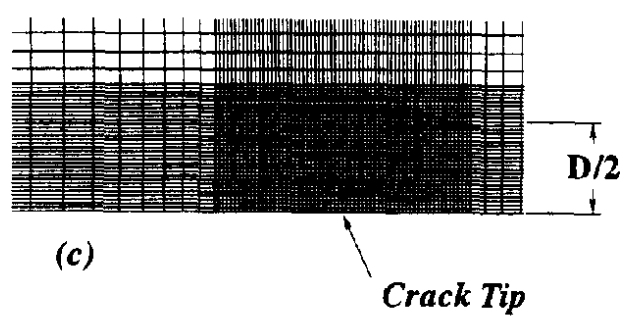

Fig. 4. Finite element mesh used to model steady-state crack growth for the geometry depicted in Fig. 3(b). "(c)" fits into "(b)" and "(b)" fits into "(a)".

with $G_{\text {tip. }}$. The results to follow have neglected this consideration. For continued crack growth, i.e. for $G_{\text {lif }}$ to remain equal to $2_{\gamma \text { inc }}, G_{\text {far }}$ will have to continue to increase, leading to an "R-curve" type of behavior. A plastic zone will continue to evolve until some steady state is achieved, after which, a constant ratio $G_{\text {iar }} / G_{\text {ip }}$ will be attained, corresponding to a uniform crack velocity and an unchanging plastic zone shape. It is this steady-state configuration that we model using the finite element method, and we assume that "small-scale yielding" conditions prevail so that the external mode I loading is uniquely characterized by $G_{\text {far }}$. Dimensional analysis then requires that the (inverse of the) shielding ratio is given by

$$
\frac{G_{\mathrm{tip}}}{G_{\mathrm{far}}}=g\left(\frac{E G_{\mathrm{far}}}{\sigma_{f}^{2} D}\right),
$$

where the function $g$, obtained directly from the finite element calculation, depends additionally on $N$ and weakly on $v$, as well as very weakly on $\sigma_{n} / E$, with that 
latter dependence manifesting itself only within the finite (versus "small") strain formulation.

An additional output quantity of interest from the FEM solution is $\sigma_{\max }$, the maximum equivalent stress in the plastic zone (defined as $\sqrt{3 s_{i j} s_{i j} / 2}$, where $s_{i j}$ are components of the stress deviator tensor). Again. dimensional analysis dictates that

$$
\frac{\sigma_{\mathrm{max}}}{\sigma_{0}}=f\left(\frac{E G_{\mathrm{far}}}{\sigma_{0}^{2} D}\right),
$$

where $f$ again depends on $N$ and weakly on $v$ and $\sigma_{0} / E$. The final step in this approach is to extract $D$, that is, to regard it as an output of the model, rather than an input, by forcing it to be consistent with $\sigma_{\max }$ via the relation discussed in equation (4) and in the following section.

The steady-state, moving crack problem is directly analyzed via the Modified Boundary Layer formulation, which involves determination of stresses within the yielding and wake zones by updating through integration in the negative crack-growth direction. In such a manner, the stress-strain history of a material point is obtained. The functions of interest, $f(\eta)$ and $g(\eta)$, are shown in Fig. 5. More details pertaining to this FEM formulation may be found in Ref. [32]. In order to obtain accurate solutions, it is imperative that the mesh is fine enough to resolve stress variation within the elastic strip, while at the same time large enough to capture the complete geometry, including the fully developed plastic zone. Naturally, as the ratio $E G_{\text {far }} / \sigma_{0}^{2} D$, which is proportional to the ratio of overall plastic zone size to $D$, increases, then satisfactory convergence of the FEM routine for a suitable mesh becomes difficult to obtain. This places an upper bound on the largest ratio $\gamma_{\text {int }} / \sigma_{0} b$ for which we provide results.

\section{THE DETERMINATION OF SELF-CONSISTENT SOLUTIONS}

The maximum stress, $\sigma_{\max }$, from the aforementioned continuum model must be made consistent

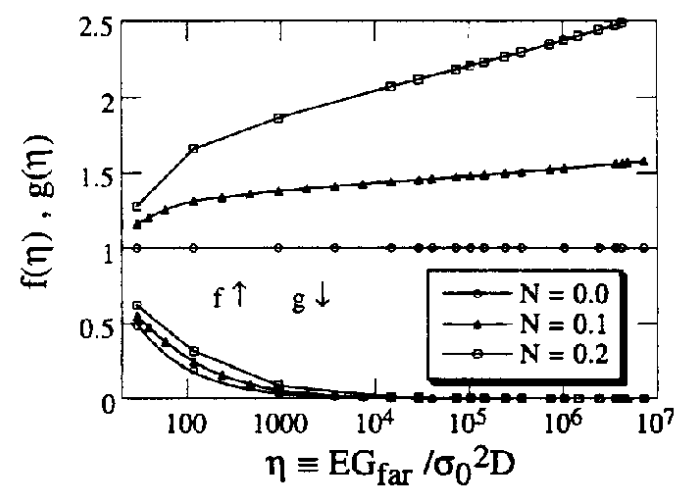

Fig. 5. Output functions $f(\eta)$ and $g(\eta)$ from FEM calculations. In all cases, $E / \sigma_{0}=1000$ and $v=0.3$; there is weak dependence on these quantities. with the stress associated with a dislocation arrangement having a characteristic barrier spacing $D$. Work hardening results when dislocations move. interact, and change their distribution and density, which generally gives rise to an increase in the flow stress. Taylor [18] first recognized that the flow stress is related to the stress required to force two dislocations on parallel slip planes of spacing $L$ past each other against their elastic interaction, which leads to the following relation:

$$
\tau_{\text {flow }}=\frac{\alpha \mu b}{L}
$$

where $\tau_{\text {fow }}$ is the resolved shear stress on the slip plane required for the barrier to be overcome and $\alpha$ is a constant of order 0.1 . It is known, however, that dislocations do not interact as simple pairs and that they form a complex entangled network. Further, in later stages of work hardening they typically arrange themselves into highly-packed regions or "walls", resulting in elastic cells of effectively negligible dislocation density. The incorporation of these aspects into theories which can explain how the evolving dislocation arrangement changes with increasing stresses and strains has been undertaken by several researchers in the past three decades, notably Kuhlmann-Wilsdorf [33], Mughrabi [34], and more recently Argon et al. [35-37]. It has been broadly recognized that an equation of the above form remains valid for these more complex dislocation arrangements where, in the range of cell structures at large strains, $L$ may be identified with $D_{\text {cell }}$ (the characteristic cell dimension) and $\alpha$ then lies in the range 5-10 for metals, and in the range 25-80 for ionic crystals and oxides [38]. Also, throughout the entire range of plastic flow studied. it appears that $L$ can be identified with the nominal dislocation spacing $1 / \sqrt{\rho_{\text {disl }}}$, regardless of whether the actual dislocation distribution is relatively homogeneous or clustered into cells, and then $\alpha$ is in the range $0.3-0.7$ $[39,40]$. When cells do form, their size seems to scale with $1 / \sqrt{\rho_{\text {disl }}}$ and be $10-20$ times larger.

In order to properly express equation (11) in terms of a macroscopic Mises equivalent tensile flow stress associated with a polycrystal (keeping in mind the isotropic behavior on larger length scales that the finite element model is designed to model), $\tau_{\text {flow }}$ must be multiplied by the Taylor factor, $M$. Recall that

$$
\sigma_{\text {fou }}=M \tau_{\text {flow }},
$$

where $M \approx 3.06$ for f.c.c. and b.c.c. crystals [41]. Combining equations (11) and (12), and noting $E=2 \mu(1+v)$, the tensile flow stress can be written as

$$
\sigma_{\text {flow }}=\frac{M \alpha E b}{2 D(1+\nu)}=\frac{\alpha_{1} E b}{D},
$$

where $\alpha_{t}$ is the analog for $\alpha$, appropriate for tensile quantities $\left(\alpha_{1} \approx 7 \alpha / 6\right)$. Finally, including a 
temperature-dependent term $\sigma^{*}(T)$ gives the form used in Section 2 of this paper

$$
\sigma_{\text {flow }}=\frac{x \cdot E b}{D}+\sigma^{*}(T) .
$$

The final stage of the model involves calculating the particular shielding ratio and cell size $D$ that make $\sigma_{\text {flow }}$ identify with $\sigma_{\max }$, the maximum $\sigma_{\text {eft }}$ from the FEM model. Doing so gives

$$
\sigma_{0} f\left(\frac{E G_{\mathrm{far}}}{\sigma_{0}^{2} D}\right)=\frac{\alpha_{1} E b}{D}+\sigma^{*}
$$

but note that the first term on the right-hand side of equation (15) can be broken up into several dimensionless groups

$$
\begin{aligned}
\sigma_{0} f\left(\frac{E G_{\mathrm{far}}}{\sigma_{0}^{2} D}\right) & \\
= & \left(\frac{G_{\text {tip }}}{G_{\text {lar }}}\right)\left(\frac{b \sigma_{\mathrm{r}}}{G_{\text {lip }}}\right)\left(\frac{E G_{\mathrm{far}}}{\sigma_{0}^{2} D}\right) \alpha_{\mathrm{l}} \sigma_{0}+\sigma^{*} .
\end{aligned}
$$

Thus, we are left with

$$
f(\eta)=\alpha_{1} g(\eta) \eta \frac{b \sigma_{u}}{G_{\text {tip }}}+\sigma^{*} i \sigma_{i},
$$

where $\eta$ is shorthand for $E G_{\text {ar }} / \sigma_{0}^{2} D$. After slight rearrangement, with $G_{\text {เіp }}$ replaced by its $G$ riffith value, $2 \gamma$ int, we arrive at

$$
\frac{\eta g(\eta)}{2\left[f(\eta)-\sigma^{*} / \sigma_{0}\right]}=\frac{\gamma_{\text {int }}}{\sigma_{\hat{\imath} 1} x_{\mathrm{l}} b}
$$

where the $\eta$ which solves this equation yields the self-consistent value of $D$ that is sought. It is important to bear in mind that the FEM model can be, and is, completely solved for a range of values of $\eta$. The value that solves the problem, however, is the one that satisfies equation (18). Once the proper value of $\eta$ is determined (we refer to it as $\eta_{0}$ ), equations (9). (10) and (14) can again be used to obtain the output quantities of interest:

$$
\begin{aligned}
& \frac{G_{\mathrm{far}}}{G_{\mathrm{ti} \mathrm{r}}}=\frac{1}{g\left(\eta_{\mathrm{u}}\right)} \equiv f_{1}\left(\frac{\ddot{\mathrm{imt}}_{\mathrm{in}}}{\sigma_{\mathrm{l}} x_{\mathrm{i}} b}: N, \frac{\sigma_{0}}{E}, \frac{\sigma^{*}}{\sigma_{0}}\right) \\
& \frac{D \sigma_{0}}{x_{1} b E}=\frac{1}{f\left(\eta_{0}\right)} \equiv f_{2}\left(\frac{\gamma_{m i}}{\sigma_{11} x_{1} b} ; N, \frac{\sigma_{0}}{E}, \frac{\sigma^{*}}{\sigma_{0}}\right) \\
& \frac{\sigma_{\max }}{\sigma_{0}}=f\left(\eta_{0}\right) \equiv f_{1}\left(\frac{\gamma_{\mathrm{mt}}}{\sigma_{n} \alpha_{1} b} ; N, \frac{\sigma_{0}}{E}, \frac{\sigma^{*}}{\sigma_{0}}\right)
\end{aligned}
$$

To summarize the principal implication of this section, the shielding ratio $G_{\mathrm{far}} / G_{\mathrm{tip}}$ and the elastic spacing $D$ are primarily dependent on the material parameter, $\gamma_{\text {in: }} / \sigma_{0} b$

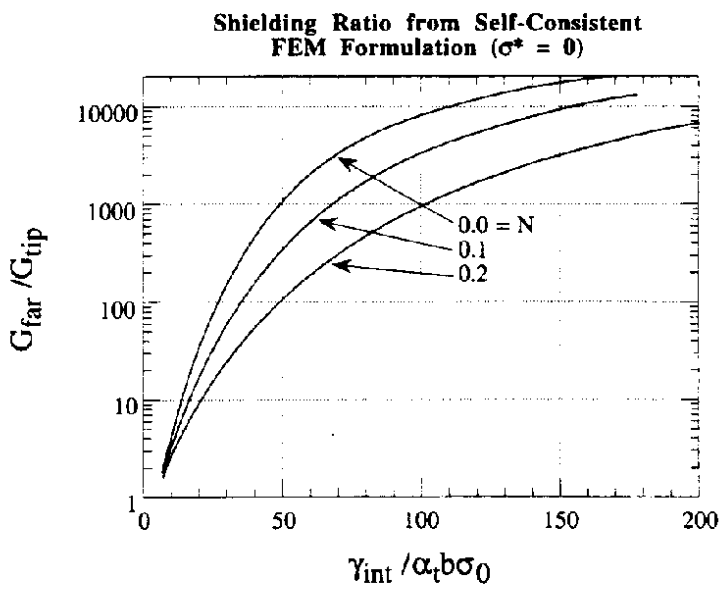

Fig. 6. Shielding ratio necessary for steady crack growth $\left(\sigma^{*}=0\right)$

\section{RESULTS AND CONCLUDING REMARKS}

Results for shielding ratio. $D$ and $\sigma_{\text {max }}$ are shown in Figs $6-8$ by plotting results of equations $(19 a-c)$ for the special case $\sigma^{*}=0$. The plots are for $v=0.3$ and $\sigma_{0} / E=0.001$; we recall that there is only weak dependence of $f_{1}, f_{2}$ and $f_{3}$ on those variables. For a more direct representation of the dependence of the shielding ratio on $\gamma_{\mathrm{nn}} / \sigma_{t \mid} b$, Figs 9 and 10 show the variation of $G_{\text {far }} / G_{\text {crit }}$, as well as $K_{\text {far }} / K_{\text {crit }}$ for specific values of $\alpha_{1}: 0.6$ in Fig. 9, which then applies for the version of the model with $D$ being nominal spacing in the random dislocation network, and 8 in Fig. 10, which applies with $D$ being cell size. Also, $N$ ranges from 0 (perfect plasticity) to 0.2 . Only the version of the model with $D$ being nominal spacing is consistent with large shielding, and our further discussion focuses on that case. Although the material parameter $\gamma_{\text {int }} / \sigma_{n} b$ varies significantly for materials in general; a rough rule of thumb is that it falls in the range $10-50$ for high-strength steels as well as metal-ceramic interfaces (the former due to high yield strengths and the latter due to values of $\gamma_{\text {in }}$ less than $\left.1 \mathrm{~J} / \mathrm{m}^{2}\right)$, and in the neighborhood of 100 for softer f.c.c. metals. The self-consistent elastic cell size

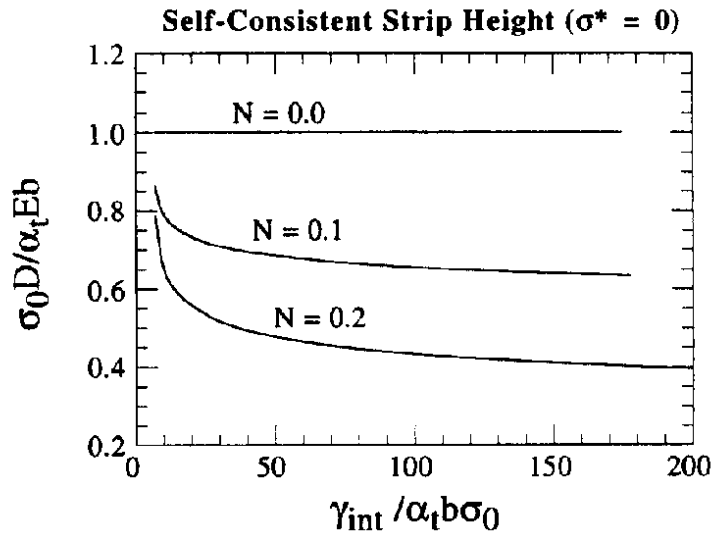

Fig. 7. Self-consistent strip height $D\left(\sigma^{*}=0\right)$. 
Maximum Effective Stress in Plastic Zone

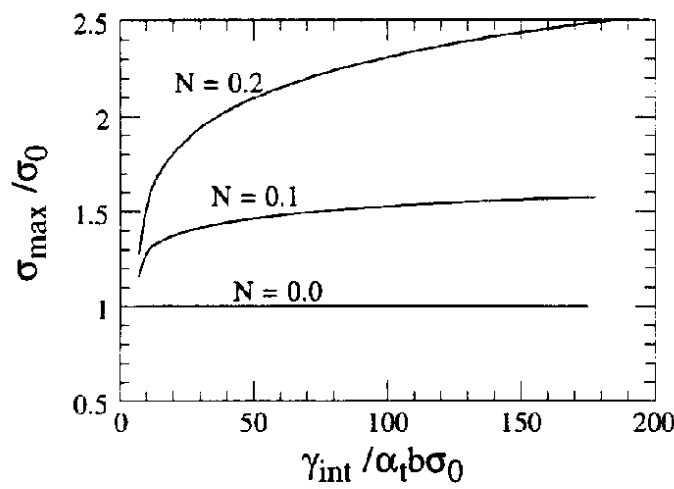

Fig. 8. Maximum effective stress in plastic zone.

ranges from 300 to $600 b$ for $x_{1}=0.6$, several times greater than those suggested by the simple calculation presented earlier in this paper, and indicates that the elastic region at least is large enough to be treated with a continuum, rather than an atomistic, theory. Furthermore, the dimension $D$ is not inconsistent with observations of dislocation arrangements in

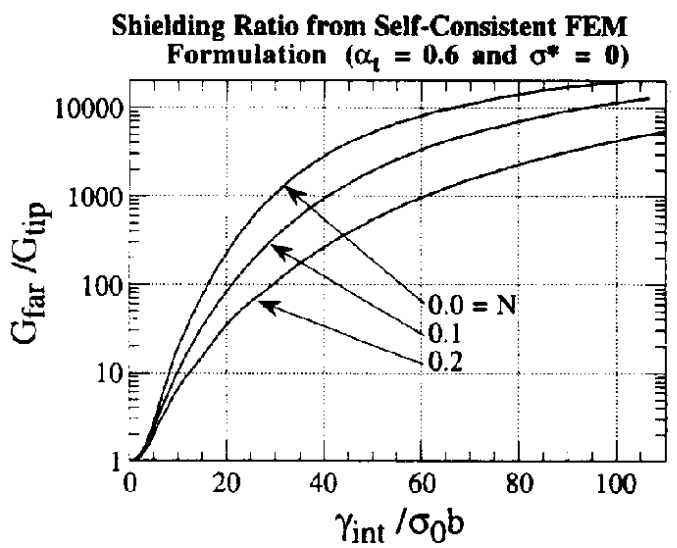

(a)

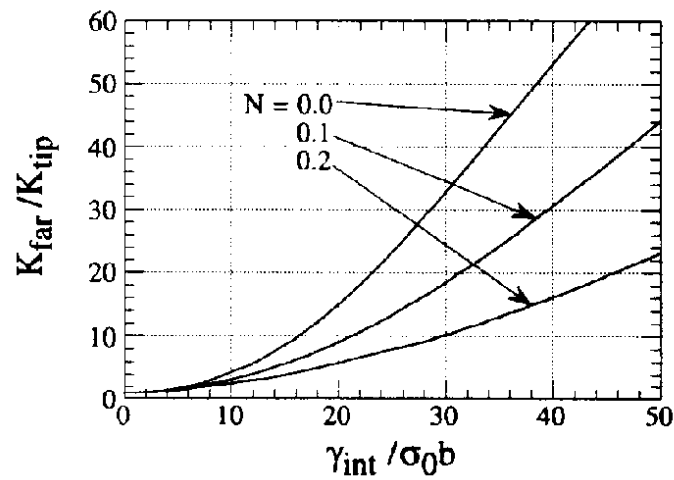

(b)

Fig. 9. Shielding ratio (expressed as the ratio $G_{\text {far }} / G_{\text {up }}$ as well as $K_{\text {far }} / K_{\text {up }}$ ) for $\alpha_{\mathrm{t}}=0.6$ (consistent with interpretation of $D$ as nominal dislocation spacing $1 / \sqrt{\rho_{\text {dist }}}$ and $\sigma^{*}=0$.

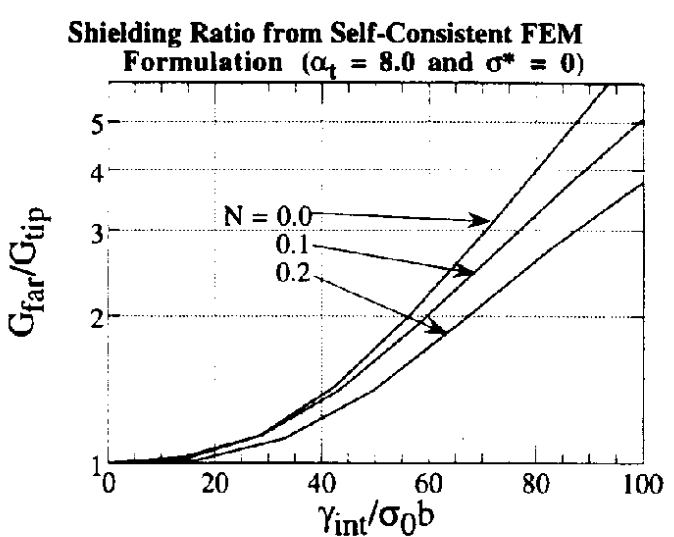

(a)

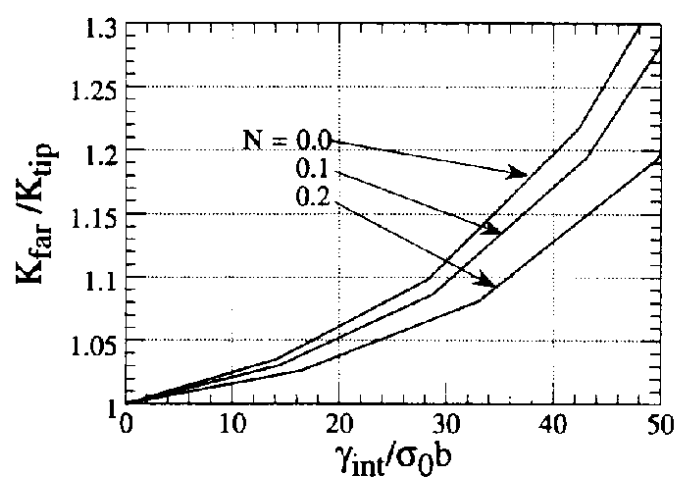

(b)

Fig. 10. Same as Fig. 9. but for $\alpha_{1}=8.0$ (consistent with interpreting $D$ as cell size) and $\sigma^{*}=0$. The shielding effect is substantially decreased.

moderately hardened metals: whereas a well-annealed crystal is expected to have a dislocation density of about $10^{11} \mathrm{~m}^{-2}$, corresponding to $D / b \approx 10,000$, a strongly hardened metal might have a density of $10^{15} \mathrm{~m}^{-2}$, corresponding to $D / b \approx 100$. The conversions here assume $D=1$ i $\rho$.

Of particular interest are the phenomenally large shielding ratios predicted by this model, as seen in Figs 9 for $\alpha_{t}=0.6$ and $\sigma^{*}=0$. The applied $G$ needed to maintain stable crack growth must range up to four orders of magnitude in excess of $G$ at the tip.

For the purposes of making contact with one of the types of experimental observations that motivated these calculations. consider the case of gold-sapphire interfaces. Although $2 \gamma_{i n t}$ for the interface has been estimated to be approximately $0.6 \mathrm{~J} / \mathrm{m}^{2}$, the actual $G$ associated with crack propagation is observed to be in excess of $200 \mathrm{~J} / \mathrm{m}^{2}$ [3]-implying a shielding ratio of at least 300 . When $\gamma_{\text {int }} / \sigma_{0} b$ decreases by a factor of 2 (which is what is approximately expected to occur when, for example, carbon is introduced into the gold-sapphire system [3]), the present calculations predict that the $G$ to maintain steady crack growth decreases by slightly less than one order of 
magnitude. Such a behavior is actually observed in the gold-sapphire system [3]. While we do not suggest that the mechanism analyzed in this paper is entirely responsible for the observed mechanical response of the gold-sapphire system, it does raise the possibility that an elastic-cell mechanism for cleavage in the presence of plastic flow is possible and may be relevant for certain systems. Although the theoretical result is not yet directly applicable to metal-ceramic interfaces (e.g. the model presented thus far is applicable to homogencous material loaded in mode 1), the result is encouraging and suggests, in a quantitative way, a formalism with which to calculate the various dissipation mechanisms in bimaterial fracture. More details pertaining to the potential application of ideas in this paper to metal-ceramic systems may be found in Ref. [42] and references therein.

Finally, let us consider the effect of $\sigma^{*}(T)$, which is critical to understanding the brittle to ductile transition with increase of $T$ predicted by the present model. Figures 11 (a)-(c) show the shielding ratio for the three hardening exponents $N=0.0,0.1$ and 0.2 , respectively, for a range of ratios $\sigma^{*} / \sigma_{0}$, necessarily less than unity since $\sigma^{*}$ is one of the components of $\sigma_{0}\left[=\sigma_{\mathrm{flc}}\right.$ of equations (4) or (14) at the start of deformation].

The effect of decrease of $T$, hence increase of $\sigma^{*}$, is twofold: first, because $\sigma^{*} / \sigma_{0}$ increases, one moves to a lower curve in Fig. 11, i.e. to less shielding. Second, because increasing $\sigma^{*}$ increases $\sigma_{0}$ equally, the value of $i \mathrm{in} / \alpha_{1} \sigma_{0} b$ is reduced, and hence one moves to the left along the horizontal axis, which further diminishes the shielding.

This is further illustrated by a set of points, $A, B$ and $C$ marked on Figs $1 \mathrm{l}(\mathrm{b})$ and (c). Point $A$ corresponds to a high enough temperature that $\sigma^{*}$ makes a negligible contribution to the yield strength, $\sigma^{*} / \sigma_{0} \approx 0$. It has been located for this illustration at $\gamma_{\text {int }} / \alpha_{\mathrm{t}} \sigma_{0} b=100$; such would, for example, correspond to $\alpha_{i}=0.6$ and to a material with $\gamma_{\mathrm{inn}} / \mu b=0.079$ (like for $x$-Fe, Table 1) and with a dislocation content such that its high-temperature yield strength is $\sigma_{0}=5 \times 10^{-4} E\left(\right.$ i.e. $\rho_{\text {dis }} \approx 10^{1.3} \mathrm{~m}^{-2}$ ). Let us write this high temperature strength $\sigma_{n}$ as $0.6 E b / D_{n}$. Then, if the temperature is reduced enough so that $\sigma^{*}$ rises to $0.6 E b / D_{11}, \sigma_{11}$ has been doubled and the conditions correspond to point $B$ in the figures, at which $\sigma^{*} / \sigma_{0}=0.5$ and $; i^{\prime} / \alpha_{1} \sigma_{0} b=50$. Thus for the case $N=0.1$. Fig. 11(b), this temperature reduction has reduced the shielding ratio $G_{\text {ar }} / 2_{i \text {; }}$ from $\sim 3000$ at point $A$ to $\sim 90$ at point $B$; for $N=0.2$. Fig. 11(c), the reduction is from $\sim 1000$ at $A$ to $\sim 50$ at $B$. These are substantial reductions and indicate a strong effect of temperature change. Point $C$ corresponds to a yet more substantial temperature reduction at which there is assumed to be a 3-fold greater increase of $\sigma^{*}$, to $1.8 \mathrm{~Eb} / D_{0}$. Then $\sigma_{0}$ is doubled again, to four times its high-temperature value, so that $\sigma^{*} / \sigma_{0}=0.75$ and $\gamma_{\mathrm{in}} / \alpha_{\mathrm{t}} \sigma_{0} b=25$; the shielding ratio drops to $\sim 5$ for $N=0.1$ and to $\sim 7$ for $N=0.2$.
Data on relatively pure Ti-gettered $\mathrm{Fe}$ and $\mathrm{Fe}-\mathrm{Co}_{0}$ alloys presented by Leslie [43] shows (his Figs 5. 13 and 26) that in the range where $\sigma_{0}$ begins to show pronounced temperature dependence, a reduction of temperature of roughly $40-50 \mathrm{~K}$ would suffice to

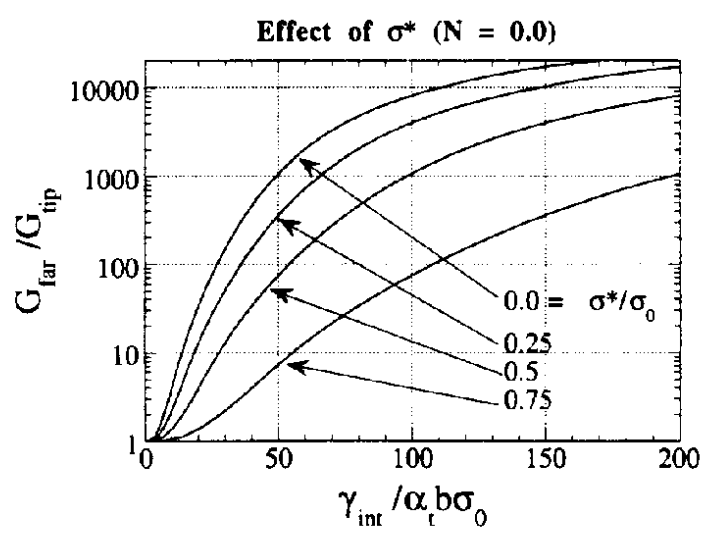

(a)

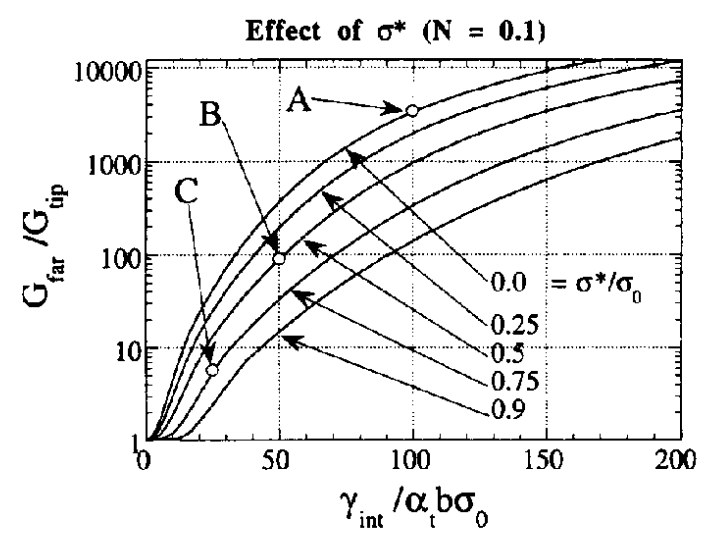

(b)

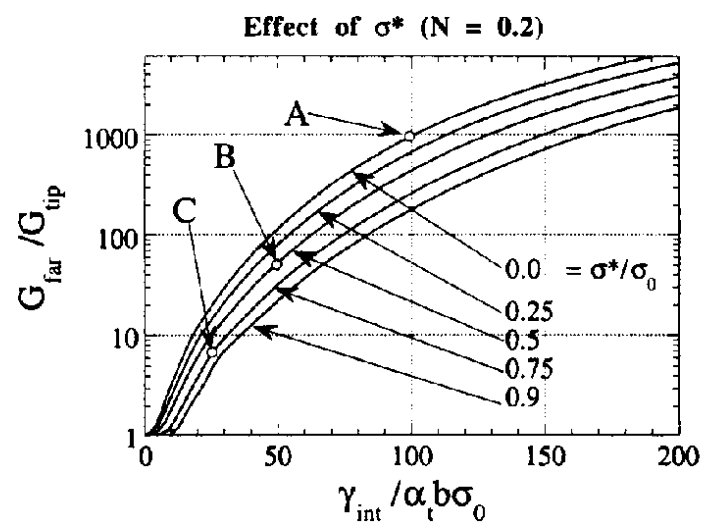

(c)

Fig. 11. Shielding ratios as a function of $\sigma^{*}$; note the associated dramatic decrease in apparent toughness with increase of $\sigma^{*}$. Transition from $A$ to $B$ corresponds to doubling of yield stress $\sigma_{0}$ through an increase of its temperature-dependent part $\sigma^{*}$ by an appropriate reduction of temperature. Transition from $B$ to $C$ corresponds to a further doubling of yield stress. See text for details 
accomplish the increase of yield strength of $\sigma^{*}$ envisioned in going from point $A$ to $B$. e.g. $\sigma^{*}$ increasing by about $5 \times 10^{-4} E$ to double the yield strength. Thus the predicted behavior does seem to have the relatively rapid variation with $T$ that one generally associates with the brittle to ductile transition in metals.

In considering the $T$ dependence of this predicted response. it is good to remember that the present model is based on the assumptions that: (a) the crack tip is non-emitting: (b) the failure mechanism is by cleavage; and (c) failure takes place under plane strain small-scale yielding conditions. While all of these assumptions may in some cases be valid at low enough $T$, one or more may fail with increasing $T$. so that the increase of $G_{\text {ar }}$ implied, e.g. by change under increasing $T$ from point $B$ to $A$ in Fig. 1l, will not necessarily be realized. This is because: (a) dislocation generation from non-emitting tips is prohibited by an energy barrier which may be breached by thermal activation at higher $T$; (b) at such high $G_{\text {far }} / G_{\text {tip }}$ values as predicted at higher $T$, failure by hole nucleation and growth may occur before the cleavage condition is met; and (c) the larger $G_{f a r}$ values may be inconsistent with small-scale yielding and plane strain constraint, so that failure occurs by general yielding.

We conclude that our present self-consistent elastic-plastic analysis of the elastic strip model leads to qualitatively, and perhaps quantitatively, sensible descriptions of cleavage cracking in metals with large densities of relatively mobile dislocations. For the future, it will be important to find more realistic representations than the elastic strip of the potentially clastic cores existing near a crack tip due to the discreteness, or granularity, of the plastic flow process. and to bridge between the present type of cleavage model and models based on viscoplastic resistance to flow.

Acknowledgements-GEB acknowledges support from the Alexander von Humboldt Foundation, the Academic Senate Committee on Research of the University of California, Santa Barbara and the MRL Program of the National Science Foundation under Award No. DMR9123048. JRR acknowledges support from the Office of Naval Research, Mechanics Division, under grants N0001490-J-1379 and 1960 to Harvard University. CFS and LX received support from the National Science Foundation under the Materials Research Group Grant DMR-9223683. Discussions with A. S. Argon, M. F. Ashby, D. R. Clarke, A. G. Evans, J. P. Hirth, J. W. Hutchinson, D. M. Lipkin, S. Dj. Mesarovic, Z. Suo, R. Thomson and J.-S. Wang are gratefully acknowledged.

\section{REFERENCES}

1. J. R. Rice, G. E. Beltz and Y. Sun, in Topics in Fracture and Fatigue (edited by A. S. Argon), p. 1. Springer, New York (1992).

2. Z. Suo. C. F. Shih and A. G. Varias, Acta metall mater. 41, 1551 (1993)

3. D. M. Lipkin, D. R. Clarke and A. G. Evans, Effect of Interfacial Segregation on Metal/Ceramic Fracture in the Gold/Sapphire System. work in progress, University of California. Santa Barbara (1996).

4. J.-S. Wang and P. M. Anderson. Acta metall. mater. 39 , 779 (1991).

5. G. E. Beltz and J.S. Wang. Acta metall mater. 40, 1675 (1992)

6. T. S. Oh, R. M. Cannon and R. O. Richie, $J$. $A m$ Ceram. Soc. 70, C352 (1987).

7. N. P. O'Dowd. M. G. Stout and C. F. Shih, Phil. Mag A. 66, 1037 (1992)

8. D. Korn. G. Elssner. H. F. Fischmeister and M. Rühle, Acta metall, mater, 40, S355 (1992).

9. Z. Suo and C. F. Shih, in Fundamentals of Metal-Marrix Composiles (edited by S. Suresh. A. Mortensen and A. Needleman). Butterworth-Heinemann. Oxford (1993).

10. P. A. Mataga. L. B. Freund and J. W. Hutchinson, J. Phys. Chem. Solids 48, 985 (1987).

11. E. W. Hart. Int. J. Solids Struct. 16, 807 (1980).

12. M. L. Jokl, J. Kameda. C. J. McMahon, Jr and V. Vitek. Metal Sci. 14, 375 (1980).

13. M. L. Jokl, V. Vitek and C. J. McMahon, Jr, Acta metall. 28, 1479 (1980).

14. H. Huang and W. W. Gerberich. Acta metall. mater. 42 639 (1994).

15. P. B. Hirsch and S. G. Roberts. Phil. Mag. A 64, 55 (1991).

16. G. E. Beltz and J. R. Rice. Acta metall mater, 40, 332 (1992).

17. G. P. Cherepanov, in Mechanics USA 1994 (edited by A. S. Kobayashi), p. S326. American Society of Mechanical Engineers, New York (1994).

18. G. 1. Taylor. Proc. R. Soc. London A 145, 362 (1934).

19. E. Orowan. in Internal Stresses in Metals and Allovs, p. 451. Institute of Metals, London (1948).

20. A. Needleman. J. Mech. Phrs. Solids 38, 289 (1990).

21. V. Tvergaard and J. W. Hutchinson. J. Mech. Phis. Solids 40, 1377 (1992)

22. V. Tvergaard and J. W. Hutchinson. J. Mech. Phys. Solids 41, 1119 (1993)

23. V. Tvergaard and J. W. Hutchinson. Phil. Mag A 70 641 (1994).

24. S. M. Ohr, Mater. Sci. Engng 72, 1 (1985).

25. J. R. Rice and R. Thomson, Phil. Mag. 29, 73 (1974).

26. R. Thomson, J. Mater. Sci. 13, 128 (1978)

27. M. F. Ashby and J. D. Embury. Scripta metall. 19, 557 (1985).

28. Y. Huang and J. R. Rice, unpublished work, Harvard University (1990).

29. V. R. Nitzsche and K. J. Hsia. Mater. Sci. Engng $A$ 176, 155 (1994)

30. J. R. Rice, in Fraciure: An Adranced Treatise, Vol. 2 (edited by H. Liebowitz), p. 191. Academic Press, New York (1968).

31. S. Dj. Mesarovic, J. Mech. Phys. Solids. in press.

32. A. G. Varias and C. F. Shih, J. Mech. Phys. Solids 41, 835 (1993).

33. D. Kuhlmann-Wilsdorf, in Work Hardening (edited by J. P. Hirth and J. Weertman), p. 97. Metallurgical Society Conferences, Vol. 46. Gordon \& Breach, New York (1968).

34. H. Mughrabi. in Constitutive Equations in Plasticity (edited by A. S. Argon), p. 199. M.I.T. Press, Cambridge, MA (1975)

35. A. S. Argon. A. K. Nigam and G. E. Padawer, Phil. Mag. 25, 1095 (1972)

36. F. Prinz and A. S. Argon. Physica status solidi (a) 57, 741 (1980).

37. A. S. Argon and P. Haasen, Acta metall mater. 41, 3289 (1993).

38. J.-P. Poirier, Creep of Crystals: High Temperature Deformation Processes in Metals, Ceramics, and 
Minerais. Cambridge University Press, Cambridge (1985).

39. S. J. Basinski and Z. S. Basinski. in Dislocations in Solids (edited by F. R. N. Nabarro), Vol 4, p. 261 North Holland. New York (1979).

40. T. E. Mitchell. in Progress in Applied Materials Research (edited by E. G. Stanford, J. H. Fearon and
W. J. McGonnagle). Vol. 6, p. 117. Heywood, London (1964).

41. R. J. Asaro, in Adrances in Applied Mechanics, Vol. 23, p. I. Academic Press. New York (1993).

42. D. M. Lipkin and G. E. Beltz. Acta mater. 44, 1287 (1996).

43. W. C. Leslie, Metall. Trans. 3, 5 (1972). 